\title{
OBSERVACCÓES PALINOLÓGICAS SOBRE ALGUNS SEDIMENTOS DO GONDVANA INFERIOR DA BACIA DO PARANÁ, BRASIL
}

\author{
DHARANI SUNDARAM
}

Aluno de Pós-Graduação, IGUSP

\section{INTRODUÇÃO}

Os sedimentos neopaleozóicos da Bacia do Paraná, Brasil, estão sendo estudados sistematicamente do ponto de vista palinológico dentro de um dos programas do projeto no 42 do IGCP. Numerosas amostras têm sido palinologicamente analisadas. Até o momento, apenas 10 amostras foram selecionadas para ilustrar o conteúdo palinológico delas. Elas foram quantitativa e qualitativamente estudadas em detalhe, para observar-se o comportamento da composição palinológica nos diferentes horizontes estratigráficos. Para esse fim, foram selecionadas amostras do Subgrupo Itararé e Formação Rio Bonito. Duas assembléias palinológicas distintas foram observadas e são discutidas. Um estudo comparativo com outras palinozonas conhecidas de continentes gondvânicos é aqui tentado.

\section{MATERIAIS E MÉTODOS}

O material do Subgrupo Itararé é proveniente das áreas de Buri e Cesário Lange, no Estado de São Paulo, enquanto o da Formação Rio Bonito é originário dos Estados de Paraná e Santa Catarina, no Sul do Brasil. As amostras pertencem a coleção do Departamento de Paleontologia e Estratigrafia, I.G. - U.S.P. A lo- calização dos pontos de origem das amostras são exibidas na fig. I.

Nenhuma nova técnica da maceração foi desenvolvida uma vez que esses materiais já se encontravam processados, por pesquisadores anteriores, pelas técnicas de maceração convencionais e lâminas delas já estavam preparadas. Apenas fotomicrografias foram tiradas para estudos de detalhe e comparação de palinomorfos. 


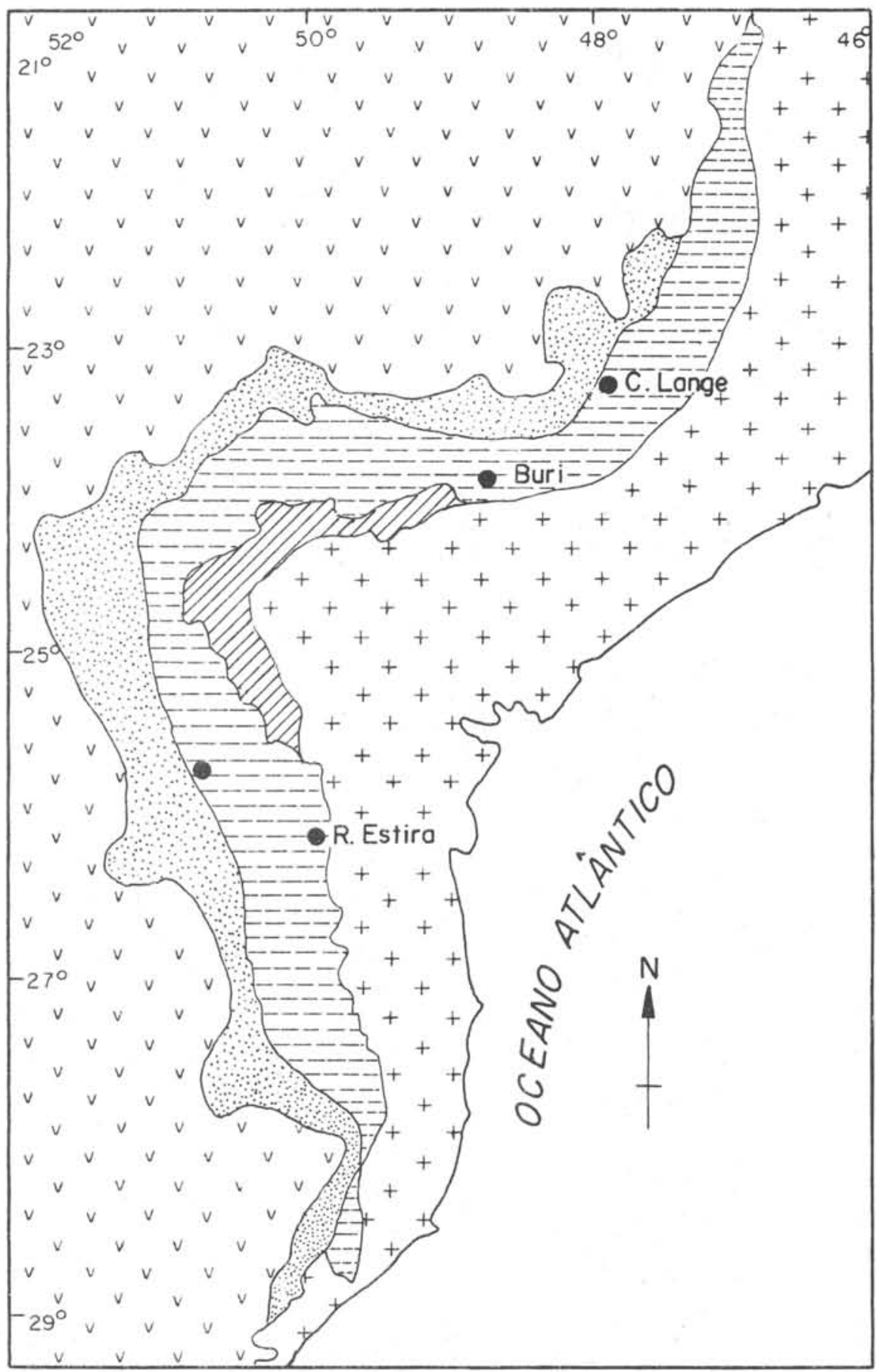

\section{LEGENDA}

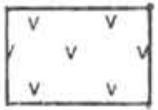
Gr. São Bento
+Bauru

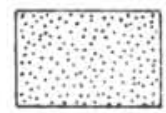

Gr. Pos. Dois

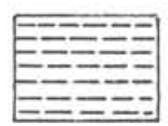

Gr. Tubarão

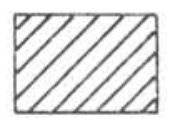

Gr. Paraná

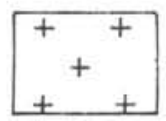

Precambriano

Localizoção de ornostras

Escala: $1: 5.000,000$

Fig. I-MAPA DE LOCALIZAÇÃO 


\section{GEOLOGIA GERAL}

Em linhas gerais, a seqüência geológica da Bacia do Paraná é tida como se segue, segundo autores anteriores:

GRUPO SUBGRUPO

PASSA DOIS

TUBARĀO

Guatá

Itararé

\section{ANALISE MIOFLORAL}

As amostras revelam a presença de uma rica assembléia mioflorística representada por todos os grupos palinomórficos maiores tais como Triletes, Monoletes, Monosaccites, Disaccites, Polyplicates etc. Os gêneros mais freqüentes referentes a esse estudo são discutidos abaixo.

Estudos qualitativos e quantitativos têm sido aplicados nessas amostras e o quadro palinológico obtido está na histograma o que torna fácil visualizar os resultados (fig. 2).

Numa visão mais ampla, os palinomorfos podem ser agrupados em duas zonas mioflorísticas distintas que informalmente têm sido designadas Palinozona A e B. As características mais importantes dessas palinozonas são discutidas abaixo.

A Palinozona A é dominada pelos Triletes especialmente as formas cinguladas e zonadas. Estes são representados por gêneros tais como Vallatisporites, Krauselisporites, Cingulatisporites, Cirratriradites, Acanthotriletes etc. Estes constituem cerca de $70-80 \%$ da composição total da assembléia. Os monossacados ocupam o segundo lugar, constituindo aproximadamente 20\%. Estão representados por Cannanoropollis, Plicatipollenites, Parasaccites. Monoletes constituem 5\%. Os disaccites são raros. Junto com outras formas completam os $5 \%$ restantes. A distribuição da freqüência é mostrada no histograma da fig. 2.

A Assembléia acima é bem observada nas amostras do Subgrupo Itararé. Alguns dos esporomorfos desta palinozona são apresentados na Estampa I.

A Palinozona B apresenta grande quantidade de palimorfos do grupo Disaccites. Aqui os dissacados não estriados são mais comuns que os estriados. Os mais comuns são Scheuringipollenites, Platysaccus Faunipollenites etc. Os estriados são Striatopodocarpites, Protohaploxypinus etc. Vittatina também é observado nessas amostras. Os dissacados atingem $60-65 \%$ enquanto os triletes que estão em quntidades subordinadas chegam apenas até $15-20 \%$. Monoletes e outros constituem $5 \%$ cada um. A abundância relativa dos tipos mais freqüentes é evidenciada na fig. 2 .

As amostras da Formaçāo Rio Bonito caracterizam esta palinozona. Alguns esporomorfos desta zona são exibidos na Estampa 3.

\section{ESTUDO COMPARATIVO}

Em seqüências sedimentares neopalezóicas de outros continentes gondvânicos, palinozonas bem distintas têm sido reconhecidas, após estudos detalhados. A partir disso, colunas palinoestratigráficas têm sido levantadas. Assim um estudo comparativo com essas palinozonas enriqueceria o conhecimento a respeito das aqui tratadas.

Este trabalho é uma tentativa preliminar e apenas com mais estudos detalhados poder-seia esclarecer esse assunto. Mas algumas generalizações amplas podem ser feitas na atual conjuntura.

$\mathrm{Na}$ África do Sul, Hart $(1969,1971)$ reconheceu quatro zonas chamadas Camerati, Cingulati, Zonati e Striatiti. Comparando as palinozonas aqui propostas com as sulafricanas percebe-se uma nítida ausência de palinozona $\mathrm{A}$, entre as últimas. Palinozona B pode ser comparada com a palinozona Zonati visto que há muitos polinomorfos comuns entre ambas.

$\mathrm{Na}$ India, 5 palinozonas têm sido observadas. Elas são Talchir, Karharbari, Barakar, Barren Measures e Raniganj. Bharadwaj (1966, $1974,1975)$ tem detalhado melhor essas palinozonas, Venkatachala (1972) tem representado graficamente a abundância relativa dos palimorfos típicos nessas zonas. Aqui também a palinozona $\mathrm{A}$ não encontra equivalente. A Palinozona B é correspondente a assembléia mioflorístíca Barakar.

Uma grande quantidade de dados palinológicos tem sido obtida a partir de sedimentos neopaleozóico da Austrália. Vários palinólogos 
têm trabalhado e fornecido detalhes. Recentemente, Kemp et allii (1977) reconheceram oito unidades palinológicas informais designadas Unidades I-VIII. Estas distribuem-se em um intervalo de tempo aproximadamente equivalente a Missouriano e Guadalupiano. A unidade I parece ser similar a palinozona $\mathrm{A}$, mas há uma
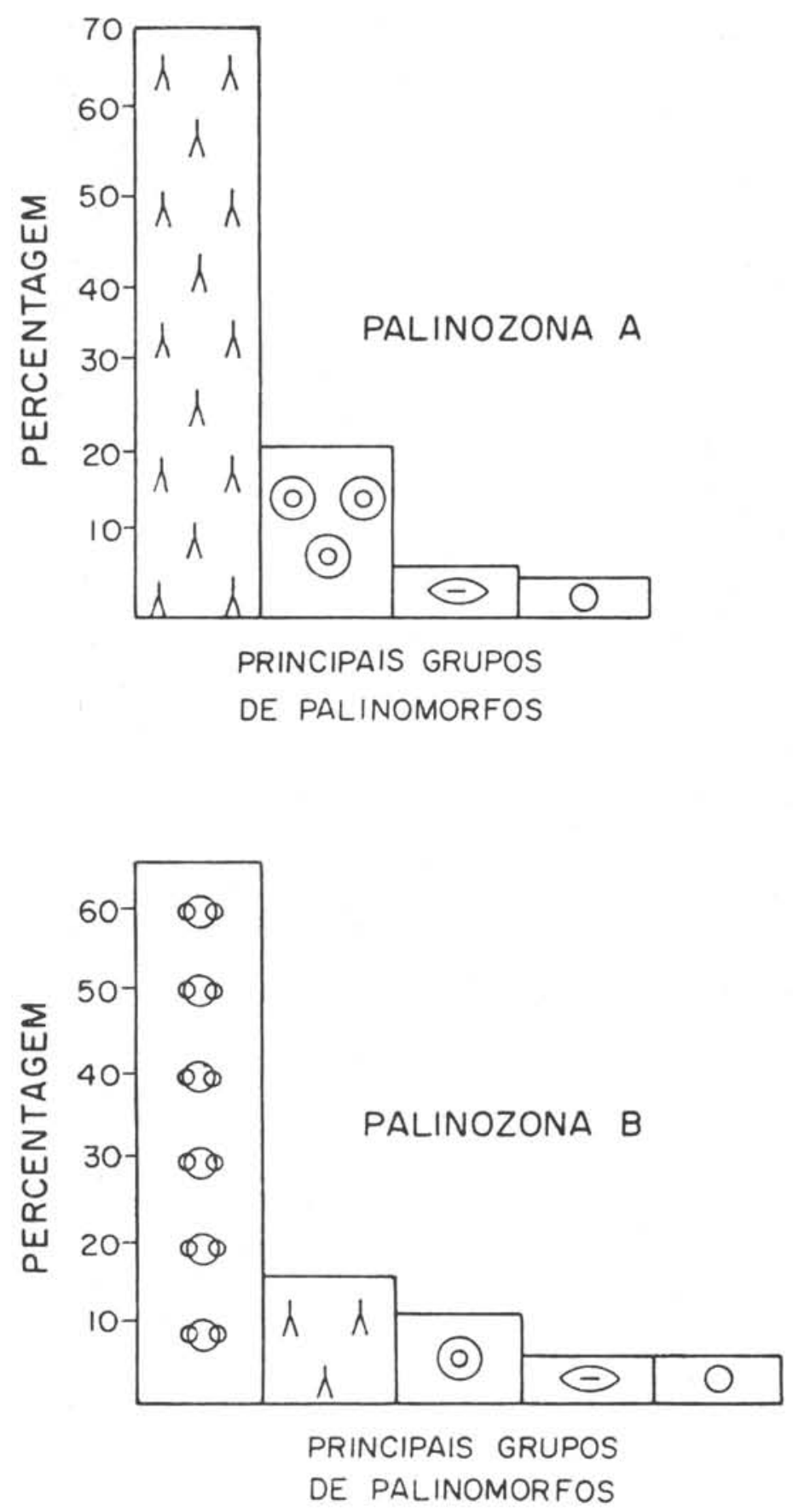

Fig. 2 - Frequência dos principais grupos de palinomorfos. diferença na pobreza relativa de monossacados nesta última. Contudo a maioria dos triletes parece ser comum a ambas. $\mathrm{A}$ unidade $\mathrm{V}$ pode ser comparada com a Palinozona B. Aqui também há diferença na abundância relativa dos palinomorfos observados. Mas de uma maneira geral ainda configuram-se como boa comparação.
LEGENDA

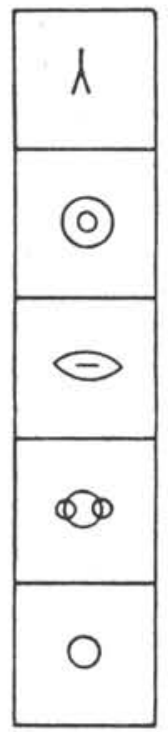

TRILETES

MONOSSACADOS

MONOLETES

BISSACADOS

OUTROS 
Palinologicamente a Bacia do Paraná está pouco trabalhada, contudo, outras informações palinológicas podem ser obtidas das bacias adjacentes tais como Chaco-Paraná e Tarija. Além disso, trabalhos anteriores foram dirigidos para os megásporos na Bacia do Paraná. Referências podem ser vistas nos trabalhos de Pant e Srivastava (1965), Triwari \& Navale (1967), Daemon e Quadros (1970), Kemp (1975). Arai (1977) forneceu maiores informações sobre esta bacia.

Recentemente, Azcuy (1976) sintetizou toda informação palinológica possivel sobre a América do Sul e estabeleceu cinco palinozonas considerando detalhadamente seu conteúdo miofloristico.

A zona I parece ser em muitos aspectos similar à Palinozona $\mathrm{A}$. Mas a primeira tem comparativamente maior quantidade de Triletes que a última. Além disso, outros elementos associados não estão bem representados na primeira.

Semeihantemente a zona $\mathrm{V}$ pode ser methor comparada com a Palinozona B. A tabela anexa mostra a correlação entre essas várias palinozonas. (Tab. I).

\section{DISCUSSÃO}

Pode ser observado que uma rica assembléia mioflorística é obtida de sedimentos neopaleozóicos da bacia do Paraná, Brasil. Estudo detalhado até o nivel específico trará mais luz fornecendo dados adicionais.

A Palinozona A é peculiar com seu característico conteúdo miofloristico. Sua correspondente nāo é observada na India e África do Sul. Suspeita-se que seja mais antiga do que as observadas naquelas áreas gondvânicas. Essa zona parece ser pré-permiana. Além disso, essa zona tem semelhantes entre bacias adjacentes como Chaco-Paraná e Paganzo sugerindo contiguidade entre estas bacias.

A Palinozona B tem grande semelhança com palinozonas encontradas nos continentes gondvânicos. Aqui também a abundância relativa de esporomorfos é variável. Daí ser necessário cautela ao tentar-se a correlação entre continentes distantes. Trabalhos mais detalhados até o nível específico mostrarão ser de grande valor na construção da coluna palinoestratigráfica dessa bacia.

\section{AGRADECIMENTOS}

O autor deseja externar sinceros agradecimentos ao Prof. Dr. A.C. Rocha Campos sob cuja orientação este trabalho está sendo feito. Quero agradecer também à Profa. Dra. Mary E. Bernardes de Oliveira pela correção do texto.

\section{TABELA ।}

\begin{tabular}{|l|c|c|c|}
\hline \multicolumn{3}{|c|}{ COMPARAÇÕO DE PALINOZONAS DOS CONTINENTES GONDVĀNICOS } \\
\hline & AMÉRICA DO SUL & ÁFRICA DO SUL & AUSTRÁLIA \\
\hline BACIA DO PARANA & (Azcuy'76) & (Hart'71) & (Kemp et alli'77) \\
(Sundaram'79) & III & Zonati & V \\
Palinozona B & 1 & & 11 \\
\hline
\end{tabular}

ESTAMPA I - Todas as figuras aumentadas $800 \mathrm{X}$
Fig. 1. Plicatipollenites
2. Acanthotriletes
3. Vallatisporites
4. Verrucosisporites
5. Punctatisporites
6. Cristatisporites

\section{ESTAMPA 2 - Todas as figuras aumentadas $800 \times$}

Fig. 1. Cannanoropollis
2. Potonieisporites
3. Platysaccus
4. Scheuringipollenites
5. Vittatina
6. Granulatisporites
7. Faunipollenites 


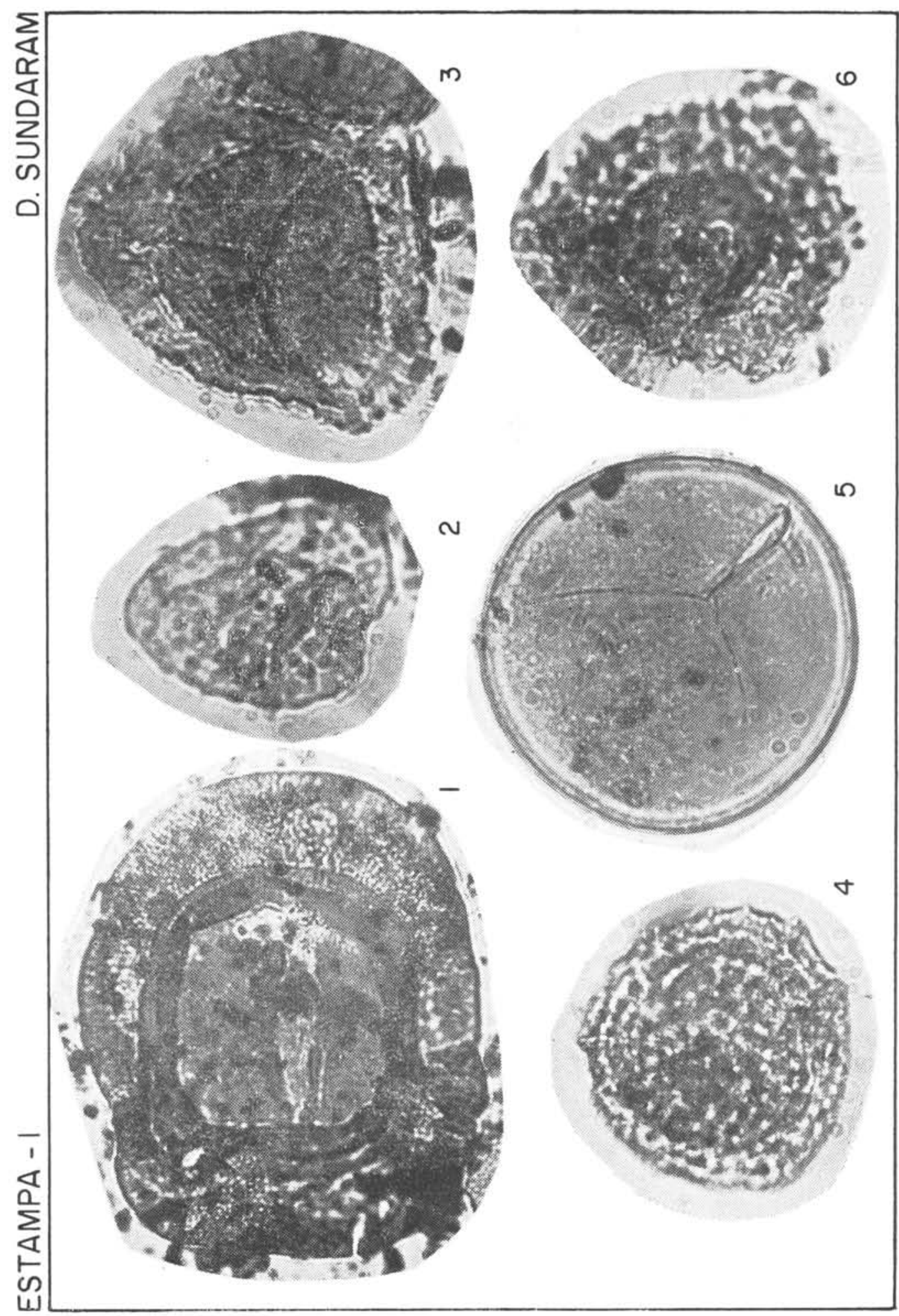




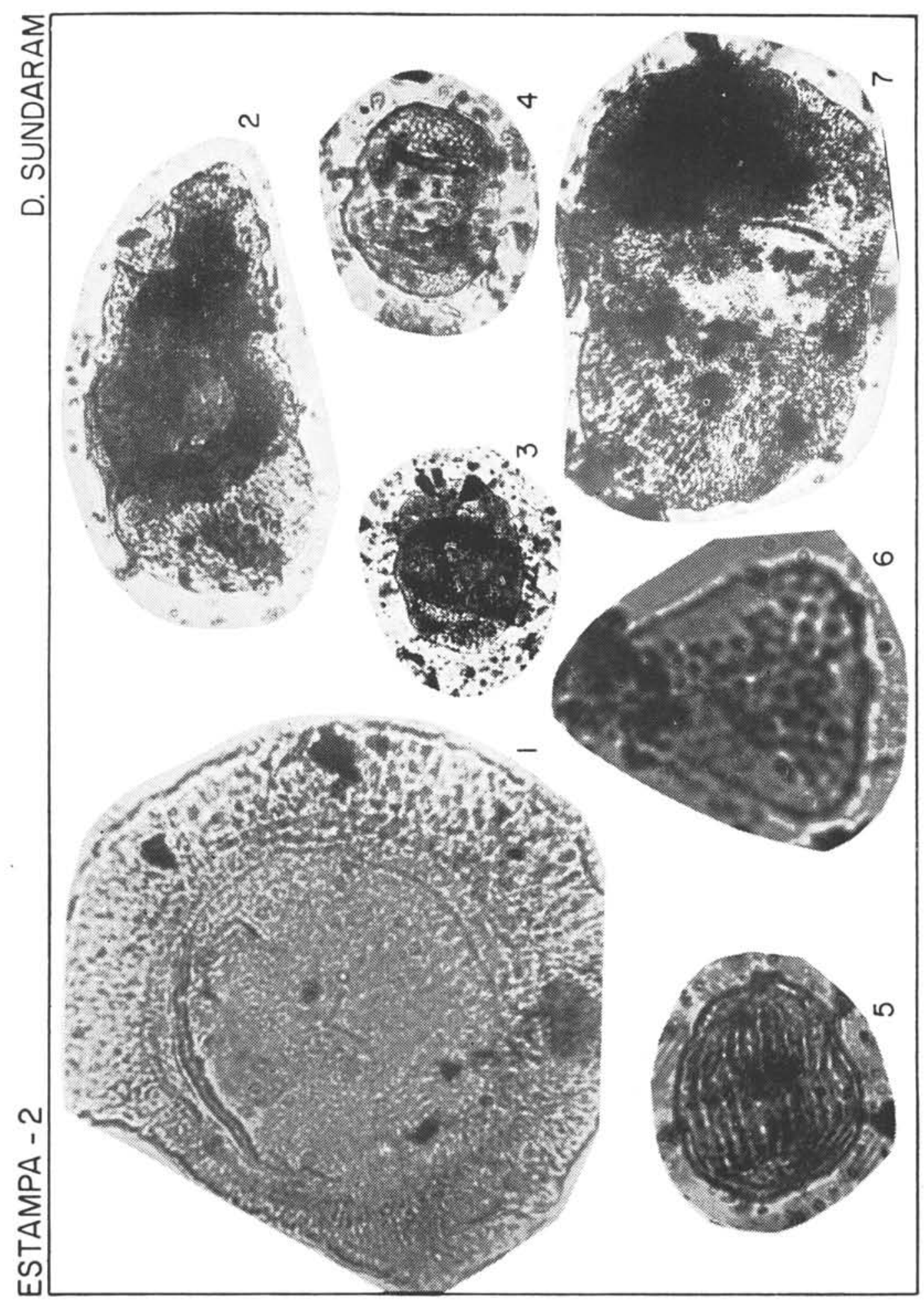




\title{
BIBLIOGRAFIA
}

ARAI, M. - 1977 - Palinologia das camadas Hulheíferas dos Estados do Paraná e São Paulo: Relatório (inédito).

AZCUY, C.L. - 1976 - A review of the early Gondwana Palynology of Argentina and South America: A review. (MS).

BHARADWAJ, D.C. - 1966 - Distribution of spores and pollen grains in the Lower Gondwana Formations of India, In: Symp. Floristics and stratigraphy. Birbal Sahni Institute of Paleobotany, Lucknow, p. 69-84.

- 1974 - Palynological subdivisions of the Damuda Series Aspects and Appraisal of Indian Palaeobotany. Birbal Sahni Institute of Palaeobotany, Lucknow, p. 392-396.

- 1975 - Palynology in the biostratigraphy and palaeoecology of Indian Gondwan Formations: The Palaeobotanis, 22: 150-57.

DAEMON, R.F. \& QUADROS, L.P. - 1970 - Bioestratigrafia do neopaleozbico da bacia do Paraná: Anais. Acad. bras. XXIV Congresso, p. 359-412.

HART, G.F. - 1969 - Stratigraphic Subdivisions and equivalents of Karroo sequence as suggested by Palynology: Gondwana Stratigraphy 2. UNESCO, Paris, p. 23-26.

p. $145-85$.

KEMP, E. - 1975 - The palynology of the Late glacial deposits of Gondwanaland. In Campbell, K.S.W. (Ed), Gondwna Geology, Australian National University Press, Canberrea, p. 397-413.

KEMP, E.M., BALME. B.E., HELBY. R.J., KYLE, R.A., PALYFORD, G. \& PRICE, P.L. - 1977 - Carboniferous and Permian Palynostratigraphy in Australia and Antarctica: A review: BMR Journal of Australian Geology and Geophysics, 2, p. 177-208.

PANT, D.D. \& SRIVASTAVA, G. - 1966 - Some Lower Gondwana miospores from Brazil: Micropalaeontology, II: $568-78$.

TIWARI, R.S. \& NAVALE, G.K.B. - 1967 - Pollen and spore assemblages in some coals of Brazil. Pollen et Spores. IX: $583-605$.

VENKATACHALA, B.S. - 1972 - Observations on some palynological contributions to Indian Stratigraphy. The Palaeobotanist, 19: 284-96.

\section{ESTUDO ESTRATIGRÃFICO E PALEOECOLOGICO EM OCORRÊNCIAS FOSSILIFFERAS DA FORMAÇĀO IRATÍ, ESTADO DE SĀO PAULO, BRASIL.}

\author{
DIANA MUSSA ${ }^{1}$ \\ RONALDO G. DE CARVALHO \\ PAULO ROBERTO DOS SANTOS ${ }^{2}$
}

(1) DGM 90 Distr. = DNPM

(2) Departamento de Paleontologia e Estratigrafia, IGUSP 